\title{
Conditioned-safe Ceremonies and a User Study of an Application to Web Authentication
}

\author{
Chris Karlof J.D. Tygar David Wagner \\ \{ckarlof, tygar, daw\}@cs.berkeley.edu \\ University of California, Berkeley
}

\begin{abstract}
We introduce the notion of a conditioned-safe ceremony. A "ceremony" is similar to the conventional notion of a protocol, except that a ceremony explicitly includes human participants. Our formulation of a conditioned-safe ceremony draws on several ideas and lessons learned from the human factors and human reliability community: forcing functions, defense in depth, and the use of human tendencies, such as rule-based decision making. We propose design principles for building conditioned-safe ceremonies and apply these principles to develop a registration ceremony for machine authentication based on email. We evaluated our email registration ceremony with a user study of 200 participants. We designed our study to be as ecologically valid as possible: we employed deception, did not use a laboratory environment, and attempted to create an experience of risk. We simulated attacks against the users and found that email registration was significantly more secure than challenge question based registration. We also found evidence that conditioning helped email registration users resist attacks, but contributed towards making challenge question users more vulnerable.
\end{abstract}

\section{Introduction}

We live in a complicated environment, and like many animals, we tend to develop automatic responses to situations we encounter more than once. Our brains tend to classify stimuli according to a few key features, and if one or more features match stimuli we have encountered in the past, we often respond mindlessly with the action that we learned was most appropriate. Psychologist Robert Cialdini calls these click-whirr responses [9]. Cialdini compares these automatic responses to pre-recorded tapes in our head, and uses "click-whirr" to evoke the sound a tape machine makes after pressing "play". As the world becomes more intricate and variable, we increasingly rely on click-whirr responses. Without click-whirr responses, we would spend most of our time appraising and analyzing mundane situations in our daily lives. Philosopher Alfred North Whitehead recognized this when he asserted "civilization advances by the extending the number of operations we can perform without thinking about them [55]."

As we become more dependent on click-whirr responses to navigate our daily lives, some have learned to exploit this behavior. Salesman, fund raisers, and con men can create situations containing the stimuli necessary to trigger the desired click-whirr response, even though less visible features may differ substantially from past situations. For example, people tend to obey a person in a uniform, regardless of whether that person has any real authority.

The designers of many current Web authentication mechanisms, such as passwords, have all but ignored this fundamental psychological phenomenon. Social engineering attacks on the Internet, such as phishing, have largely been successful because the Web is fertile ground for mimicry, and password authentication can condition users to fall for these attacks. Many users have developed a click-whirr response to login forms and will automatically enter their login credentials on any Web page that mimics a trusted site and on the surface, appears legitimate.

In response to these social engineering threats, many institutions use machine authentication, which authenticates a user's computer, in addition to password authentication, which authenticates the user herself. Since a user may use more than one computer, machine authentication systems must have a registration procedure to authorize and set authentication cookies on multiple machines. Many machine authentication systems currently deployed by financial Web sites use challenge question based registration [7, 27, 49]. A challenge question is a user-specific question to which an adversary is unlikely to be able to guess an answer, e.g., "What is the name of your favorite teacher?" [18, 32]. Registration based on challenge questions is vulnerable to man-in-the-middle (MITM) attacks [46, 61]. Since these attacks exploit similar click-whirr responses as attacks against passwords, the security benefits of challenge questions over passwords alone may be minimal. 


\begin{tabular}{|c|c|c|c|c|c|}
\hline Group & Registration method & Attack & $\begin{array}{c}\text { Warnings } \\
\text { in email? }\end{array}$ & Size & $\begin{array}{c}\text { Attack } \\
\text { successful }\end{array}$ \\
\hline \hline 1 & Challenge questions & Solicit answers & N.A. & 41 & $92.7 \%(38)$ \\
\hline 2 & Email & Forwarding & $\checkmark$ & 40 & $40.0 \%(16)$ \\
\hline 3 & Email & Forwarding & & 39 & $30.8 \%(12)$ \\
\hline 4 & Email & Cut and paste & $\checkmark$ & 40 & $47.5 \%(19)$ \\
\hline 5 & Email & Cut and paste & & 40 & $47.5 \%(19)$ \\
\hline
\end{tabular}

Table 1. Success rates of our simulated attacks against registration procedures in our user study. Users in groups 2 and 4 received contextual warnings in registration emails against our simulated attacks, but users in groups 3 and 5 did not.

\subsection{Contributions}

Since human click-whirr responses seem to be an unavoidable fact of life, we argue that authentication mechanisms for humans should be designed such that click-whirr responses reinforce their security. Towards realizing this goal, we make two contributions:

Conditioned-safe ceremonies. A ceremony is similar to the conventional notion of a network protocol, except that a ceremony explicitly includes human participants as nodes in the network, distinct from the computers and devices they use [17]. ${ }^{1}$ In this paper, we introduce the notion of a conditioned-safe ceremony. A conditioned-safe ceremony is one that deliberately conditions users to reflexively act in ways that protect them from attacks. Our formulation of a conditioned-safe ceremony draws on several ideas and lessons learned from the human factors and human reliability community: forcing functions, defense in depth, and the use of human tendencies, such as rule-based decision making. In Section 4, we propose design principles for conditioned-safe ceremonies.

A user study of an email based registration ceremony. We apply our design principles for conditioned-safe ceremonies to develop a registration ceremony for machine authentication based on email (Section 5). To evaluate our email based registration ceremony, we conducted a user study with 200 participants to compare the security of email registration to the security of registration based on challenge questions (Section 6). We simulated social engineering attacks against the users and found email based registration was significantly more secure against our attacks (Table 1). Our simulated attacks succeeded against $93 \%$ of

\footnotetext{
${ }^{1}$ The term ceremony was first coined for this purpose by Jesse Walker [17]. Communications between human nodes and other nodes in the ceremony are usually not via network connections, but instead through user interfaces, face-to-face interactions, or peripheral devices. Examples of ceremonies include password authentication and registration procedures.
}

challenge question users, but succeeded against only $41 \%$ of email users. We also found evidence that conditioning helped email registration users resist our simulated attacks, but contributed towards making challenge question users more vulnerable. We asked users to complete an exit survey after they finished the study, and we analyze the results in Sections 7 and 8.

\section{Why users are vulnerable}

Over the last decade, the success of phishing and other social engineering attacks has created a multi-million dollar underground economy [20,47]. Although it is tempting to blame the success of these attacks on the ignorance of users, researchers have offered an alternative explanation: computer security mechanisms such as passwords and browser security indicators are poorly suited for human use. Psychologists and security researchers have identified several ways in which many security mechanisms and ceremonies disregard human tendencies and condition users to make insecure decisions [1, 12, 13, 14, 24, 44, 53]:

Click-whirr responses and rule-based decision making are exploitable. Click-whirr responses are an example of more general human tendency to vastly prefer rulebased decision making over more tedious analytical approaches $[40,41]$. The theory of rule-based decision making is based on psychological studies that suggest humans tend to learn and aggressively apply problem-solving rules of the form "if (situation) then (action)" for frequently encountered situations. When a user encounters a problem in a task, she matches the most prominent cues in the environment with the calling conditions of previously learned rules to find most appropriate one to apply.

Although rule-based decision making helps us navigate the minutia of our daily lives and reserve our time and energy for tasks requiring more detailed analysis, adversaries can exploit rule-based decision making in social engineering attacks [9]. Human reliability expert James Reason ob- 
served that frequently used rules, i.e., strong rules, may be "misapplied in environment conditions that share some common features with the appropriate states, but also possess elements demanding a different set of actions [41]." In other words, a rule which has been frequently useful in the past can become a strong-but-wrong rule when the situational cues change subtly. This helps explains why phishing attacks have been so successful. Since a wide range of Web sites require a user to $\log$ in before she can do something interesting, many users have developed a rule of the form "if (login form) then (enter username/password)" and will aggressively apply it when they encounter login prompts on Web pages which on the surface appear familiar, legitimate, or trustworthy.

Browser security mechanisms condition users to satisfice. A frequently recommended defense against phishing attacks is for a user to verify a Web page's domain and SSL certificate before entering her password on that page; otherwise, she might inadvertently reveal her credentials to an attacker. However, research has shown that users often omit these checks [13, 14, 26, 29, 44, 54, 58]. Although some users ignore these indicators because they do not understand them, a more fundamental problem is that browser security indicators condition users to satisfice.

Satisficing is a decision-making strategy which means "to accept a choice or judgment as one that is good enough", i.e., one that both satisfies and suffices [42]. Checking security indicators is easy to skip because it distracts the user from her primary focus, and there are rarely any immediate visible consequences for skipping these checks or rewards for making them. Since the vast majority of a user's login attempts are probably not under attack (or at least do not obviously appear to be under attack), routinely skipping security checks and ignoring warnings seems deceivingly acceptable. Over time, users learn to quickly and instinctively perform a security task's required actions (e.g., entering their passwords) and optimize out the optional actions (e.g., checking security indicators, responding to security warnings). Once a user has become conditioned into a satisficed behavior, psychologists have found it is difficult for her to change it, even if she recognizes overwhelming evidence that her behavior is wrong [9].

Users are not good at recognizing attacks. Accidents and successful social engineering attacks share similar key characteristics: they have a similar precondition, i.e., a risky situation, and similar trigger, i.e., human error. A recurring theme in the field of human reliability and error is that users often have difficulty in recognizing risky or dangerous situations, and as a result, users may be less vigilant of their choices in these situations than they should. For example, in a review of 100 maritime shipping accidents, Wa- genaar and Groeneweg concluded: "Accidents do not occur because people gamble and lose, they occur because people do not believe that the accident that is about to occur is at all possible [50]." Also, decades of buggy software have conditioned users to expect errors, failures, and other incomprehensible system behavior, particularly with hastily developed and continually updated Web applications. Users routinely encounter warnings and errors messages, but rarely experience any immediate negative consequences for dismissing them, even during a real attack. This creates the (accurate) impression that false positives are the norm, and actual attacks are rare. These tendencies suggest that we cannot rely on users' abilities to detect social engineering attacks and respond appropriately, and we must design defenses accordingly.

\section{Machine authentication to the rescue?}

In response to the precipitous rise in social engineering attacks on the Internet, many institutions have implemented authentication ceremonies that supplement password based authentication with machine authentication, which authenticates a user's computer as opposed to the user herself. For example, one widely used approach for machine authentication is to set a persistent cookie; since the user's browser will send that cookie every time the user returns to the Web site from that computer, the Web site can recognize the user's computer. To successfully log in, the user must provide her password and the user's browser must present a valid cookie. The intention is to take the human "out of the loop" and reduce the system's dependency on humans' abilities to detect attacks. Web sites currently using machine authentication include Bank of America [7], ING Direct [27] and Vanguard [49].

The registration problem. Since users may use more than one computer, machine authentication systems must have a registration ceremony to authorize and set authentication cookies on multiple machines. Unfortunately, this additional functionality brings the human back "in the loop" and exposes machine authentication systems to an alternative attack vector. Instead of trying to steal authentication cookies directly from a user's machine, an attacker can try to subvert the registration ceremony in a way that grants the attacker a valid cookie for the user's account. Consequently, registration ceremonies must resist these kinds of bootstrapping attacks; otherwise, the security benefits of machine authentication may be minimal.

Challenge questions. Many machine authentication systems currently deployed by financial Web sites use challenge questions in their registration ceremony [7, 27, 49]. 
When a user creates her account, she provides the answers to one or more challenge questions, and when she attempts to $\log$ in from an unregistered computer, the site prompts her to answer these questions. If the answers are correct, then the site sets a persistent authentication cookie on the user's computer. For future logins from that computer, the user only needs to enter her password.

Challenge questions are vulnerable to an active man-inthe-middle (MITM) attacker spoofing the login page of the target Web site [46, 61]. When the user attempts to login via the spoofed page, the attacker forwards the user's login credentials to the legitimate Web site. Since the attacker is indistinguishable from the actual user logging from an unregistered machine, the Web site responds with challenge questions for the user. The attacker displays these questions to the user. After the user provides her answers, the attacker forwards them to the Web site and receives an authentication cookie for the user's account.

Challenge question based registration is vulnerable because, like password authentication, it disregards human tendencies and conditions users to fall for attacks. A user is most likely to resist an attack against her challenge questions if she recognizes the threat and refrains from the clickwhirr response of providing her answers. However, since the attacker's registration request is indistinguishable from the Web site's legitimate registration requests, detecting attacks is non-trivial for many users. Users must actively and carefully check browser security indicators, e.g., the URL bar and SSL certificate, to detect spoofing attacks. Many users misinterpret these indicators, and satisficing users often ignore them.

Registration based on challenge questions threatens to undermine the promise of machine authentication. Since users who are vulnerable to phishing attacks against their passwords will probably also be vulnerable to phishing attacks against their challenge questions, a registration ceremony using challenge questions is hardly more secure than using passwords alone. We need better registration ceremonies to realize the benefits of machine authentication.

\section{Conditioned-safe ceremonies}

One natural response to the weaknesses of challenge questions and passwords is to design ceremonies which try to eliminate user conditioning, click-whirr responses, and rule-based decision making. This approach is problematic. Rule-based decision making is fundamental to human behavior: it helps us complete routine tasks quickly and easily. Users may be willing to invest extra time and effort to learn a new security mechanism, but if they cannot learn how to use it efficiently, they will become frustrated and disable or circumvent the offending mechanism [22, 24, 60]. Some degree of conditioning may be necessary for the psychological acceptance of security mechanisms by users.

Since users will tend to adopt rules for completing a ceremony that minimize conscious effort, we should not fight users' tendencies to use rule-based decision making, but take advantage of these tendencies to help users resist social engineering attacks. We should prudently design ceremonies to condition rules that benefit security rather than undermine it. Towards achieving this goal, we introduce the notion of a conditioned-safe ceremony. A conditionedsafe ceremony is one that deliberately conditions users to reflexively act in ways that protect them from attacks. We propose two design principles for building conditioned-safe ceremonies:

- Conditioned-safe ceremonies should only condition safe rules, i.e., rules that are harmless to apply in the presence of an adversary.

- Conditioned-safe ceremonies should condition at least one immunizing rule, i.e., a rule which when applied during an attack causes the attack to fail. We discuss immunizing rules further in Section 4.1.

These principles also have important consequences on what conditioned-safe ceremonies should not do:

- Conditioned-safe ceremonies should not condition rules that require users to decide whether it is safe to apply them. Since many users are unreliable at recognizing risky situations, users should not need to refrain from conditioned behavior to resist attacks.

- Conditioned-safe ceremonies should not assume users will reliably perform actions that: 1) the ceremony has not conditioned her to perform, or 2) are voluntary. Satisficing users will learn to omit optional and voluntary actions, so ceremonies should not rely upon users to perform such actions.

For example, a ceremony should not condition the rule "if (legitimate looking login form) then (enter username/password)" because it causes a security failure when applied in the presence of an adversary. To determine if it is safe to apply this rule, a user must first verify the URL bar, the site's SSL certificate, and other security indicators. Burdening users with these decisions is unsatisfactory. Ideally, in a conditioned-safe ceremony, a user should be able to resist an attack even if she has no idea she is at risk and performs the same actions as she usually performs under benign conditions.

However, user behavior is unpredictable and an adversary may try to trick users into deviating from their normal, conditioned behavior in a way that causes a security failure. Conditioned-safe ceremonies need safeguards to resist these attacks. In the human reliability community, designers often introduce constraints called forcing functions to help 
prevent errors in safety-critical environments. We argue that forcing functions can also be useful for conditioned-safe ceremonies, and we discuss them further in the next section.

\subsection{Forcing functions}

A forcing function is a type of behavior-shaping constraint designed to prevent human error [38]. Forcing functions usually work by preventing a user from progressing in her task until she performs a particular action whose omission would result in a failure or accident. Because users must take this action during every instance of the task, the forcing function conditions users to always perform this action. With an effective forcing function, after a user performs the function's associated action, many mistakes become difficult or impossible to make. For example, consider the error of locking your keys in your residence. A potential forcing function in this situation is a door that can only be locked from the outside, keys in hand. This trains you to take your keys with you whenever you leave home, making it less likely you will be locked out in the future.

Forcing functions often have two benefits: 1) they help prevent errors of omission, where a user skips an important, protective step in a task, and 2) they condition correct, safe behavior, since users cannot normally proceed otherwise. To be effective, the cognitive and physical effort required to comply with a forcing function must be less than the effort required to circumvent it. Otherwise, users may routinely attempt to circumvent the forcing function, diminishing its benefits.

Since forcing functions have been useful for preventing errors in safety-critical environments, we hypothesize they can also help prevent errors during social engineering attacks. However, designing forcing functions that resist social engineering attacks is challenging. In conventional safety-critical environments, the risk elements rarely try to intentionally subvert protection mechanisms and cause errors. Designing electrical safety equipment would be a much trickier business if electricity had malicious intent. Also, deployability considerations for many ceremonies, e.g., no custom hardware, often require forcing functions to be implemented entirely in software. Software environments afford attackers many opportunities for mimicry.

One previous application of software-based forcing functions in computer security is the concept of a secure attention key (SAK). A SAK is a mandatory special key combination users must type before they can take a securitycritical action, e.g., submitting their password. On Window NT systems, users must type Control-Alt-Delete to get a login prompt. The SAK diverts control to the OS kernel, foiling any user-level spoofed login prompts. Since typing the SAK is mandatory, the hope is that users will learn to always enter the SAK before submitting their password.
Unfortunately, a simple attack against many SAKs is to induce an error of omission. On Windows NT systems, an adversary can display a spoofed login prompt and hope users skip the SAK before entering their passwords. This attack creates a conflict between two click-whirr responses: SAK systems condition users to first type the SAK, but all password systems condition users to enter their passwords when they see a login form. Whether the attack succeeds depends on which click-whirr response is stronger in a particular user.

Since social engineering attacks can often misrepresent the state of a system and create the illusion that a forcing function has already been activated or disabled, ceremonies which fail solely due to errors of omission are suboptimal. Errors of omission are easy to make and hard to detect, even during routine tasks. Research suggests that users frequently do not notice when they have omitted routine procedural steps [3], and omission errors represent one of the most common causes of human performance problems [39].

To resist social engineering attacks, we argue that conditioned-safe ceremonies need defense in depth. Designers should build conditioned-safe ceremonies that have two levels of protection: an attack should fail unless a user both omits the conditioned action required by a forcing function and makes an error of commission. We consider an error of commission to be an anomalous user action not normally used in the ceremony. If the user omits the action required by the forcing function, but does not otherwise deviate from the ceremony, an attack should fail. Likewise, if the user performs the required action, but then makes an error of commission, the attack should also fail. With this approach, the action conditioned by the forcing function acquires an immunizing quality, since after a user performs this action, subsequent errors of commission will not compromise the ceremony.

We emphasize that the conditioned action required by the forcing function must be easy for users to perform; in particular, it should easier to perform than any unsafe error of commission. Since humans have been conditioned to work around buggy software, a user may willingly make an effortless error of commission if she feels it will complete the security task and allow her to continue with her primary task.

\subsection{Analysis and discussion}

Although a designer can choose the rules conditioned by a ceremony, an attacker can affect which rules a user chooses to apply by manipulating the environmental stimuli. Research by psychologists and human reliability specialists suggests that users mainly rely on two processes to determine the most appropriate rule to apply in a given situation: similarity-matching and frequency-gambling [41]. 
With similarity-matching, a user compares the situation's environmental cues against cues contained in the calling conditions of previously learned rules. If she finds a unique match, she performs the associated action. If the environmental cues are underspecified and partially match several rules, she will tend to "gamble" in favor of the useful, high frequency candidates, i.e., the "good" rules which have been most frequently been applied in the past.

These tendencies suggest that conditioned-safe ceremonies will better resist the currently successful attack strategy of blatantly initiating a ceremony with the victim and presenting familiar environmental cues, e.g., spoofing a trusted Web site. Since a forcing function requires a user to perform the immunizing action every time (whether under attack or not), the forcing function will condition a highfrequency, "good" rule (namely, perform the immunizing action) that is likely to be routinely applied in the future - even when under attack. Mimicking a conditioned-safe ceremony becomes less advantageous to an adversary; if a user recognizes she is participating in the ceremony, she will tend to perform the conditioned, immunizing action, which thwarts attacks. This presents an attacker two options: 1) obviously initiate the ceremony and try to induce an error of commission before the user performs the immunizing action, or 2) surreptitiously initiate the ceremony and try to induce an error of commission without the user realizing she is participating in the ceremony.

If attackers resort to the first option, adversaries must prevent the human tendency to use rule-based decision making, rather than encourage it, as current attacks do. This creates a disadvantage for adversaries; preventing human tendencies is often difficult. If attackers resort to the second option, we hope adversaries will need to present unfamiliar situations to prevent users from recognizing the ceremony. Otherwise, users will tend to react with conditioned responses, i.e., apply safe rules and perform immunizing actions. This approach also disadvantages adversaries. Unfamiliar situations require additional cognitive effort to analyze and may cause feelings of suspicion and discomfort. User often reject unfamiliar experiences in favor of more familiar ones. For example, studies suggest that some users distrust phishing warnings because the familiar experience presented by the adversary appears more trustworthy $[16,58]$. Conditioned-safe ceremonies turn the tables and force adversaries to be the ones required to present an awkward and unfamiliar experience.

Limitations. We acknowledge conditioned-safe ceremonies have their limits. Adversaries may try to convince users to disable protective mechanisms or take actions outside the scope of a ceremony which violate certain security assumptions. For example, with the configuration of many current computer systems, if a user chooses to install mal- ware at any point, most ceremonies will be compromised. However, if we can design ceremonies that are so unproductive to attack directly that adversaries must resort to convincing users to install malware, it would be a tremendous step forward.

\section{A conditioned-safe registration ceremony using email}

In this section, we describe a conditioned-safe registration ceremony for machine authentication using email. When a user attempts to log in from an unregistered computer, the Web site sends her an email containing a singleuse HTTPS URL with an unpredictable component, for example:

$$
\text { https://www.xyz.com/reg.php?url_id=r }
$$

where $r$ is a 160 bit random number generated by the Web site. $^{2}$ We call this URL a registration link. The email includes instructions to click on the link. The Web site stores $r$ in a database, along with the associated user ID, an expiration time, and validity bit. When the user clicks on the registration link, if $r$ is still valid and has not expired, the Web site sets a persistent authentication cookie on the user's computer and invalidates $r$. A user only needs to complete this ceremony once at each computer. For subsequent logins, she only needs to complete any supplementary login procedures, e.g., enter her username and password. Several researchers have proposed using email in a similar way to help initialize authentication credentials [2, 6, 21, 23, 48].

Security analysis. Consider the threat model of a phisher, an adversary which lures unsuspecting Internet users to a Web site posing as a trustworthy business with which the users have a relationship [4]. We assume a phisher has the following capabilities: 1) complete control of a Web server with a public IP address; 2) ability to send communications such as emails and instant messages to potential victims; and 3) ability to mount application-layer man-in-the-middle attacks [5, 36, 51, 52], representing a legitimate server to the victim and proxying input from the victim to the real server as needed.

Against the phishing threat model, we argue email registration follows the principles of a conditioned-safe ceremony we propose in Section 4. The phisher can solicit the user's login name and password, but since the phisher's computer is unregistered, the site will not allow it to access the user's account without submitting a valid registration link. The attacker can trick the Web site to send the user

\footnotetext{
${ }^{2}$ We assume the user has previously given the Web site her email address, e.g., during the account creation process.
} 
a registration link, but to compromise the ceremony, an attacker must steal and use a registration link before the user submits it herself. ${ }^{3}$

The registration link acts as forcing function. Under normal conditions, a user must click on the link to proceed. Although there may be other ways of submitting the link, e.g., by copying and pasting it in the URL bar, clicking generally requires less effort, and sites can embed the URL of the link in an HTML element to make the alternatives more difficult. Also, clicking on the registration link is an immunizing action; after the Web site invalidates the link, it is useless to an attacker.

Email based registration has defense in depth. To compromise the ceremony an attacker must 1) prevent the user from clicking on the link (i.e., omit the forcing function action), and 2) trick the user into revealing the link (i.e., make an error of commission). One possible attack strategy would be to inform the user that she must register her computer, but due to "technical problems" she should not click on the link and instead give the link to the attacker. We identify two compelling and straightforward attacks of this kind: 1) ask the user to copy and paste the registration link into a text box, or 2) ask the user to forward the registration email to an address with a similar domain name as the target site. If a user does not notice the attacker's instructions and believes she is participating in the "normal" registration ceremony, we hypothesize she will likely resist these attacks. Email registration conditions users to click on the registration link, and if she clicks the link, she will resist the attack.

Alternatively, if the user notices the attacker's instructions to deviate from the ceremony, she will be safe as long as she clicks on the link before doing anything else. Since: 1) the Web site has conditioned the user to click on the registration link; 2) the credible repercussions of clicking on link are probably limited; and 3) clicking on the registration link is arguably at least as easy as complying with the instructions, the theory of rule-based decision making suggests that users will first tend to try clicking on the registration link before complying with the adversary's instructions.

The key question is the strength of users' tendencies to click the registration link rather than comply with the adversary's instructions. To help answer this question, we conducted a user study to estimate how well email registration helps users resist social engineering attacks against it. In the next section, we describe this study.

\footnotetext{
${ }^{3}$ We do not consider attacks which enable adversaries to steal users' authentication cookies after they have been set, e.g., cross-site scripting attacks or malware. This problem is orthogonal to registration and requires a different solution.
}

\section{A user study of email registration}

In this section, we describe a user study we conducted to compare the security of email registration to the security of registration using challenge questions. Our study simulated man-in-the-middle (MITM) social engineering attacks against users of each of the ceremonies. Our hypothesis is that email registration is significantly more resistant to MITM social engineering attacks than registration using challenge questions. We previously published a workshop paper describing the design of our study, but it did not present any results [33].

\subsection{Study overview}

Ecological validity is crucial: our study must realistically simulate experiences users have in the real world. This raises a number of challenges, including:

- Simulating the experience of risk for users without crossing ethical boundaries [31].

- Limiting the effect of demand characteristics, where users try to guess the study's purpose and change their behavior, perhaps unintentionally.

- Minimizing the impact of perceived authority figures during the study $[25,37]$.

- Determining an appropriate physical location for the experiment which minimizes any unrealistic influences on users.

Our study addressed these issues in two ways: 1) we did not use a laboratory, and 2) we employed deception to hide the study's true purpose. We recruited users remotely, and during the consent process, we told users that our experiment aimed to determine how well individuals can predict high grossing movies. We told each user she will log in to our Web site over a seven day period and make a prediction of what she thinks will be the top three highest grossing movies each day. Each user logged in from her "natural habitat": from her own computer, from anywhere, and at any time she wished. We show a screenshot of our interface in Figure 1.

Each user received $\$ 20$ as base compensation, and we rewarded her up to an additional $\$ 3$ per prediction depending on the accuracy of her predictions. We told each user that she must make seven predictions to complete the experiment, so the total maximum a user could receive is $\$ 41$.

We simulated the experience of risk by giving users password-protected accounts at our Web site and creating an illusion that money they "win" during the study was "banked" in these accounts. We paid users at the end of the study via PayPal and solicited each user's PayPal email 


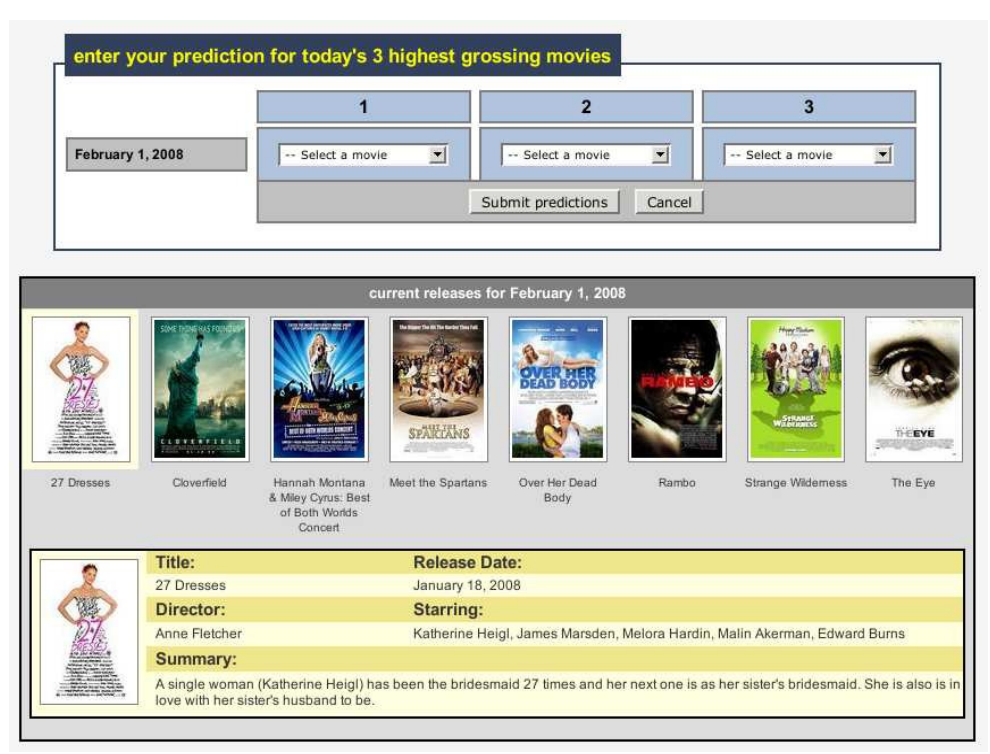

Figure 1. User interface for making predictions at our study Web site.

address at the beginning of the study. ${ }^{4}$ To help suggest that there was a risk that the user's compensation could be stolen if her account was hijacked, we provided an "account management" page which allowed the user to change the PayPal email address associated with her account.

Although we told users they must make seven predictions to complete the study, after each user made her fifth prediction, we simulated a MITM social engineering attack against her the next time she logged in. After she entered her username and password, we redirected her to an "attack" server. We discuss the simulated attacks in Section 6.4. After the simulated attack, we debriefed each user about the true purpose of the study and requested her reconsent for the use of her data.

\subsection{Recruitment}

We recruited users through the Experimental Social Science Laboratory (Xlab) at UC Berkeley. The Xlab is an interdisciplinary facility that supports UC Berkeley investigators in running behavioral and social science experiments. Members of the UC Berkeley community (i.e., students, staff, etc.) register with the Xlab over the Web and receive solicitations to participate in experiments via email. One limitation of this recruitment method is that our user pool was primarily composed of university students and staff and may not be representative of the general population. Our experiment used only native English speakers, and the subject pool included approximately 1950 eligible users.

\footnotetext{
${ }^{4}$ Although we did not verify each user's PayPal account was valid at the start of the study, each user explicitly acknowledged she either had an account or was willing to get one.
}

We contacted 225 randomly selected users in April 2008 through the Xlab. Interested users signed up through the Xlab's system and received instructions to visit our study Web site. We did meet any of the users in person. 208 users signed up for our study, and we assigned them round-robin to 5 study groups. One group used challenge questions for registration and the other four groups used different variants of email registration links. We discuss the email registration groups further in Section 6.4.2. We excluded the results of 8 users and give details in Section 7.1. We show a summary of the user groups and their sizes in Table 2.

\subsection{Registration procedures}

Each user created an account at our site, with a username and password. We also asked for the user's email address and PayPal email address, if different. After a user entered her username and password on her first login, we redirected her to a page that informed her that she must register her computer before she could use it to access her account. If the user chose to register her computer, we redirected her to the registration page. If she was in the challenge question group, we prompted her to set up her challenge questions. She selected two questions and provided answers. After confirming the answers, she entered her account and proceeded with her first prediction.

If she was part of an email registration group, then she saw a page informing her that she had been sent a registration email and must click on the link labeled "Click on this secure link to register your computer". After clicking on the link, she entered her account and made a prediction. We sent registration emails in HTML format, but also 


\begin{tabular}{|c|c|c|c|c|}
\hline Group & Size & Registration method & Attack description & Warnings in email? \\
\hline \hline 1 & 41 & Challenge questions & Solicit answers & N.A. \\
\hline 2 & 40 & Email & Forward email to attacker & $\checkmark$ \\
\hline 3 & 39 & Email & Forward email to attacker & \\
\hline 4 & 40 & Email & Copy and paste link into text box & $\checkmark$ \\
\hline 5 & 40 & Email & Copy and paste link into text box & \\
\hline
\end{tabular}

Table 2. Summary of study groups.

included a plain text alternative (using the multipart/ alternative content type) for users who had HTML viewing disabled in their email clients. We embedded the same registration link in both parts, but included a distinguishing parameter in the link to record whether the user was presented with the HTML or plain text version of the email. We discuss how we used this information in Section 6.4.2. Screenshots of registration emails are shown in Figures 3(a) and 3(b).

Both registration procedures set an HTTP cookie and a Flash Local Shared Object on the user's computer to indicate the computer is registered. For subsequent login attempts, we first prompted the user for her username and password. If the username and password were valid, our server checked if the user's computer was registered for that username. If she was logging in from a registered computer, then we redirected her to her account. If she was logging in from a computer we didn't recognize, then we prompted her to answer her challenge questions (Figure 2(a)) or sent her a new registration link to click on, depending on the user's group.

\subsection{Simulated attacks}

\subsubsection{Challenge questions: Group 1}

For the challenge question group, the attack attempted to convince users to answer their challenge questions by presenting the page shown in Figure 2(b). This is essentially the same page users saw when they answered their challenge questions under "normal" conditions, but with the warning and informative text removed. ${ }^{5}$ This attack: 1) is straightforward, 2) closely mimics the legitimate registration process, and 3) was previously disclosed in the security community as a major weakness of challenge questions $[46,61]$.

\subsubsection{Email: Groups 2-5}

For the email groups, we simulated the two attacks we identified in Section 5: the copy and paste attack and the for-

\footnotetext{
${ }^{5}$ Even if users select their challenge questions from a pool of possible questions, an attacker can easily determine a particular user's questions by relaying communications between the legitimate site and the user [46, 61].
}

warding attack. The copy and paste attack asked the user to copy the registration link into a text box, and the forwarding attack asked the user to forward the registration email to an address with a similar domain name as our study site. We simulated the forwarding attack against groups 2 and 3, and simulated the cut and paste attack against groups 4 and 5 .

We chose these attacks because we believed they are the most compelling and straightforward attacks that we could ethically implement. Another potentially effective attack would be to try to hijack each user's email account, but we did not believe this attack was ethical. We leave other attacks as a subject for future work.

For both attacks, the attack page first told the user that "because of problems with our email registration system" she should not click on the link in the email she received. For the copy and paste attack, the attack page presented a text box with a "submit" button and instructed the user to copy and paste the registration link into the box. For the forwarding attack, it instructed the user to forward the email to the attacker's email address. We show screenshots of the attack pages in Figures 4(a) and 4(b).

These attacks also presented pictorial versions of the instructions, with a screenshot of how the registration link appears in the email. To maximize the effectiveness of this picture, we gave the attacker the advantage of knowing the distribution of HTML and plain text registration emails previously viewed by the user during the study (see Section 6.3). The attack displayed the pictorial instructions corresponding to the majority; in case of a tie we displayed a screenshot of the HTML version.

\subsubsection{Warnings}

Some Web sites warn users about safe security practices, e.g., how to resist phishing attacks against challenge questions. Although these warnings are sometimes useful, they will likely be absent during an attack, when a user needs them the most. Email registration has the advantage of being able to include advisory information and contextual warnings in each registration email. To measure the effectiveness of these kinds of warnings, we subdivided the email groups into two groups: those who received warnings in registration emails (groups 2 and 4) and those who did not (groups 3 and 5). Everyone saw these warnings on le- 


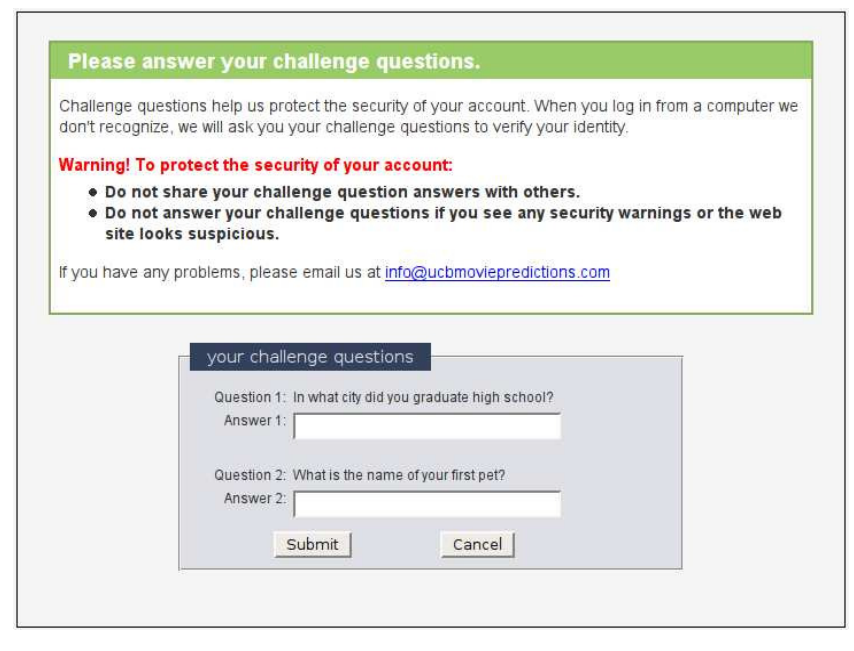

(a) User interface for answering challenge questions.

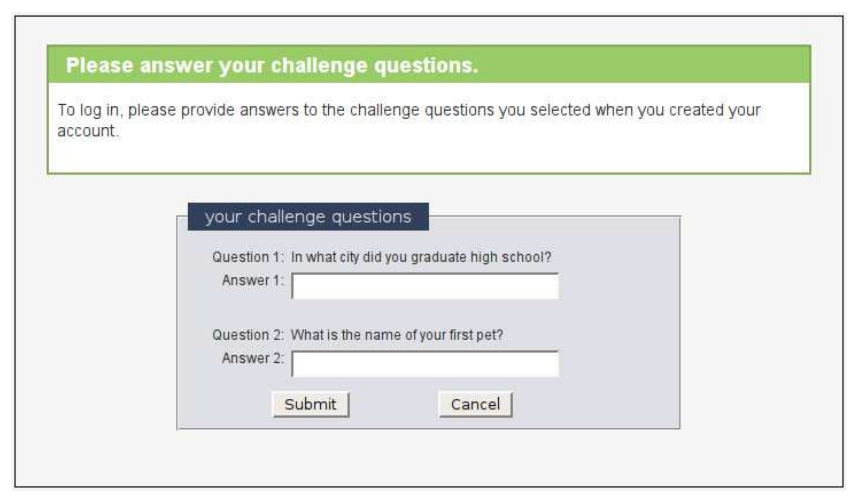

(b) Screenshot of the attack against challenge questions.

Figure 2. Normal challenge questions interface vs. simulated attack instructions.

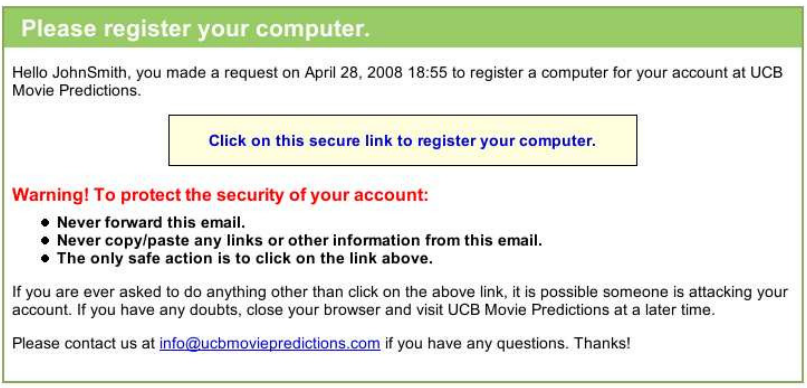

(a) HTML registration email with warnings.

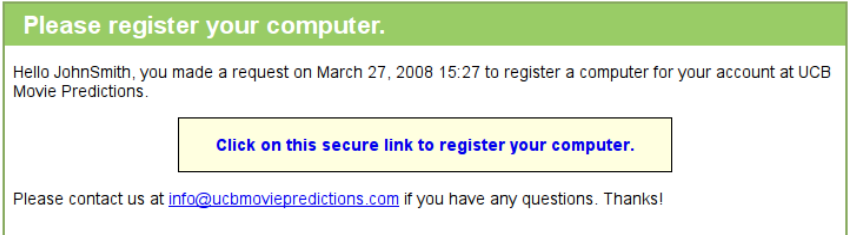

(b) HTML registration email without warnings.

Figure 3. Registration emails.

gitimate registration pages. A screenshot of these warnings is shown in Figure 3(a). ${ }^{6}$ Group 1 users also received warnings about safe practices when answering their challenge questions, but we only showed group 1 users these warnings during legitimate registrations. Group 1 users never received warnings in email.

\subsubsection{Attack success metrics}

If a group 1 user answered her challenge questions correctly on the attack page, we considered the attack a success and ended the experiment. We assumed an attacker could dis-

\footnotetext{
${ }^{6}$ These warnings specifically warned against the attacks we simulated. Although in the real world it may not be feasible to concisely warn users against all the possible attacks, a site can certainly warn users against the most successful or common attacks they have observed in the past.
}

tinguish between correct and incorrect answers (e.g., by relaying the user's responses in real time to the legitimate site), so if a user entered an incorrect answer, the attacker prompted her again.

If a group 2-5 user clicked on the registration link first, then we considered the attack a failure. ${ }^{7}$ If the user forwarded the email or submitted the link first, then we considered the attack a success. Either way, we ended the experiment for the user.

\footnotetext{
${ }^{7}$ These attacks actually simulated network level MITM attacks. Such attackers might be able to intercept registration links and steal any registration tokens stored on the user's computer. There are various proposals that can help protect registration links and cookies against stronger adversaries [10, 28, 34], but we do not discuss the details here. Regardless, the results of this study are applicable to a wide variety of social engineering attacks, including phishing.
} 


\section{Please check your email at JohnSmith@gmail.com.}

To register this computer, please look for a registration email from UCB Movie Predictions at JohnSmith@gmail.com.

1. Because of problems with our email registration system, please DO NOT CLICK ON THE LINK in the email you receive.

2. Instead, please forward the registration email to support@ucb-moviepredictions.com and then log in again.

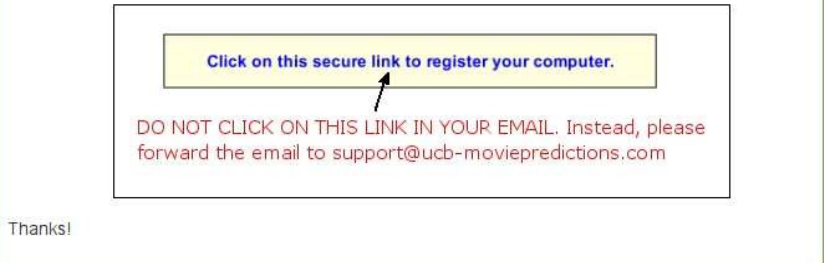

(a) Screenshot of the forwarding attack against email registration.

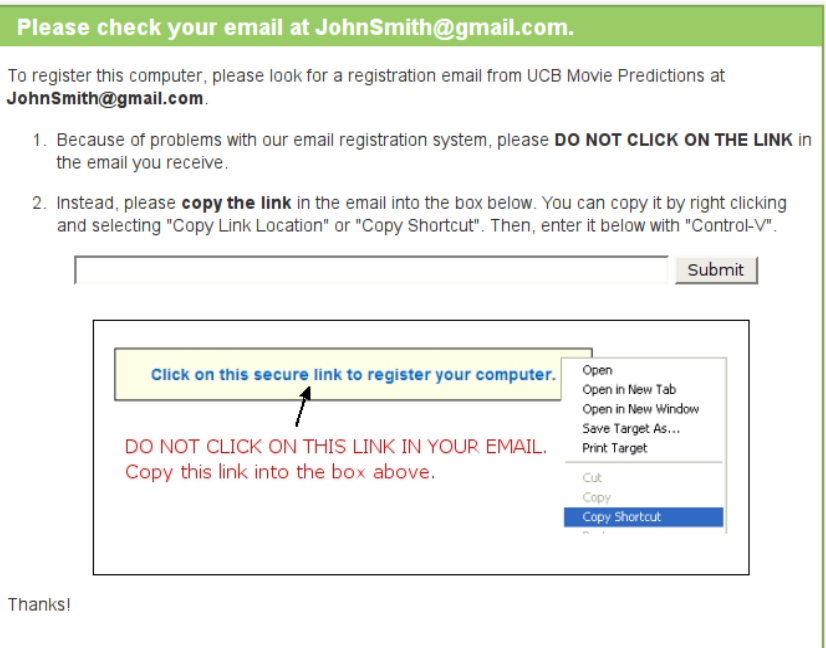

(b) Screenshot of the cut and paste attack against email registration.

Figure 4. Our simulated attacks against email registration.

For all users, attempts to navigate to other parts of the site redirected the user back to the attack page. If the user resisted the attack for 30 minutes, then on her next login, the experiment ended and we considered the attack a failure. The attack pages for groups 1,4 , and 5 contained a Javascript key logger, in case a user began to answer her challenge questions or entered the link, but then changed her mind and did not submit. If our key logger detected this, we considered the attack a success.

\subsection{Debriefing and exit survey}

After a user completed the study, we redirected her to a page that debriefed her about the true purpose of the experiment and explained the reasons for deception. The debriefing page explained the concept of machine authentication and the different ways of registering computers. We then obtained reconsent from each user. If a user reconsented, we redirected her to an exit survey.

Our exit survey started with general demographic questions such as gender, age range, and occupational area. The second section of the survey collected information on the user's general computing background, attitudes, and habits. The final section asked more specific questions about the user's experiences during the study. We discuss these questions in Section 7.

\subsection{Ethics}

Our simulated attacks were ethical. The risk to users during the attacks was minimal. We only used data from users who explicitly reconsented after a debriefing on the true nature of the study. The study protocol described here was approved by the UC Berkeley's Institutional Review Board on human experimentation.

To protect users' privacy, all connections to our web site used SSL. We purchased a certificate for our domain which is accepted by major Web browsers. In a real world attack, an attacker would most likely not be able to obtain a valid certificate for the target site. To avoid certificate warnings, an attacker would probably use HTTP rather than HTTPS to host the attack page. However, to protect users' privacy, our simulated attack page used SSL. Since our hypothesis is that email registration is more secure than challenge questions, we had to ensure that our imperfect simulation did not bias the results against challenge questions. Our solution was to maximize the benefits of SSL for the challenge question users and minimize the benefits of SSL for the email registration users. In particular, we conservatively assumed that our simulated adversary attacking email registration had obtained a valid certificate for the target domain while our simulated adversary attacking challenge question based registration had not obtained a valid certificate. Group 2-5 users did not see certificate warnings during the attack, but group 1 users did. We implemented this by redirecting group 1 users to a different Apache instance (at port 8090) with a self-signed certificate, while group 2-5 users continued to use the original Apache instance in "attack mode". This implies the "attack" domain shown in the URL bar for group 1 users included a port number, but the "attack" domain for group 2-5 users did not. 


\section{Study results}

\subsection{User demographics}

One email registration user did not complete the study, and one email registration user did not reconsent. Due to a misconfiguration, our server did not send registration emails to 6 users during the simulated attack. We excluded these users' data from our results, leaving 200 users total.

$56 \%$ of users self-reported themselves as female, $41 \%$ reported themselves as male, and 3\% did not respond. Our users were mostly young: $91 \%$ reported themselves as 1825 years old and $89 \%$ reported themselves as undergraduate students. Among students, the mix of major areas was diverse. The largest group was physical sciences (i.e., chemistry, physics, biology, etc.), accounting for $25 \%$ of users, and the second largest was economics and business, accounting for $20 \%$ of users. Computer science and engineering accounted for $1.5 \%$ and $7.5 \%$ of users, respectively.

Most users reported using Windows (69\%) and Mac OS $(25 \%)$ as their primary operating systems, and most users reported using Firefox (70\%), Internet Explorer (11\%), and Safari (10\%) as their primary Web browsers. This differs significantly from recent statistics, which report Windows as having $91 \%$ market share and Internet Explorer as having $72 \%$ market share [8].

$78 \%$ of users reported using a Web browser at least 10 hours a week, and $70 \%$ of users reported they have conducted financial transactions online for at least a year. Types of online transactions reported include PayPal (55\%), banking $(80 \%)$, investing (12\%), auctions (42\%), and shopping $(80 \%)$.

\subsection{Success of simulated attacks}

We summarize our results by group number in Table 1 . Our attack succeeded against $92.7 \%$ of challenge question users and $41.5 \%$ of email users. This difference is statistically significant ( $p<0.001$, Fisher's exact test). The cut and paste attack was slightly more effective than the forwarding attack (47\% vs. $40 \%$ with warnings, and $47 \%$ vs. $31 \%$ without warnings), but we did not find this difference significant ( $p=0.65$ with warnings, and $p=0.17$ without warnings; Fisher's exact test). We found no significant correlations between attack success and the demographics we reported in Section 7.1. In particular, we found no evidence that frequent browser use, previous experience with online financial transactions, or a technical undergraduate major area helped users resist our attacks.

\subsection{Efficacy of our warnings}

We found no evidence that including warnings in registration emails helped users resist our attack. To evalu- ate the effectiveness of our contextual email warnings, we compared the attack success rates of group 2 vs. group 3 (forwarding attacks, with and without warnings, resp.), and group 4 vs. group 5 (cut and paste attacks, with and without warnings, resp.). For the forwarding attack, $40 \%$ of group 2 users were vulnerable, and $31 \%$ of group 3 users were vulnerable ( $p=0.48$ for Fisher's exact test). For the cut and paste attack, $47 \%$ of users in both group 4 and group 5 were vulnerable ( $p=1$ for Fisher's exact test).

During the exit survey, we showed each user a screenshot of the warning corresponding to her study group (Section 6.4.3) and asked her "Do you remember seeing the above warning at any point during the study?", and if yes, "describe how it affected your decisions (if at all) while interacting with the study Web site." Table 3 summarizes the number of yes/no responses. For group 2 and 4 users, who received warnings in registration emails, $31 \%$ reported that they did not remember the warning. Among group 3 and 5 users, who only received warnings on the study Web page, $68 \%$ did not remember the warning. This difference is statistically significant ( $p<0.001$ for Fisher's exact test). $66 \%$ of challenge question users also did not remember the warning.

We found no evidence that users who recalled seeing our warnings were more likely to resist our attack. Of the 191 users responding to the warning recall question, 85 remembered seeing our warning and 106 did not (see Table 4). Among the users who remembered seeing our warning, $45 \%$ were vulnerable, and among the users who did not remember seeing our warning, $56 \%$ were vulnerable. This different is not statistically significant ( $p=.147$ for Fisher's exact test). We found no statistically significant difference within groups, either.

Among the users who remembered seeing the warning, Table 5 summarizes the self-reported effects that the warnings had on those users. Of the 85 users who remembered our warnings, only 10 users' responses $(12 \%)$ indicated that our warnings helped them resist our attack. 38 of these users $(45 \%)$ indicated that the warnings had little or no impact on their decisions. 4 users $(5 \%)$ indicated that the warning slightly influenced their decision making during the attack, but they ultimately followed the attack instructions. 7 users (8\%) mentioned that the warnings made them "feel safer" at our site or be more careful in general. The responses of 11 users were inconclusive or did not clearly fit in one of these categories.

\subsection{User suspicion of our attacks}

To gauge users' suspicion during our simulated attack, we asked users "During your interactions with UCB Movie Predictions, did you ever see something which looked suspicious or dangerous?" and "describe what your reaction 


\begin{tabular}{|c|c|c|c|c|}
\hline & Warnings & \multicolumn{3}{|c|}{ User remembered seeing our warning? } \\
Group & in email? & No & Yes & No response \\
\hline \hline 1 & N/A & $65.9 \%(27)$ & $31.7 \%(13)$ & $2.4 \%(1)$ \\
\hline 2 & $\checkmark$ & $25.0 \%(10)$ & $62.5 \%(25)$ & $12.5 \%(5)$ \\
\hline 3 & & $59.0 \%(23)$ & $41.0 \%(16)$ & $0.0 \%(0)$ \\
\hline 4 & $\checkmark$ & $37.5 \%(15)$ & $57.5 \%(23)$ & $5.0 \%(2)$ \\
\hline 5 & & $77.5 \%(31)$ & $20.0 \%(8)$ & $2.5 \%(1)$ \\
\hline
\end{tabular}

Table 3. Number of users who reported remembering seeing our warnings.

\begin{tabular}{|c|c|c|c|}
\hline & Safe & Vulnerable & Total \\
\hline \hline Users who remembered seeing our warnings & $55.3 \%(47)$ & $44.7 \%(38)$ & 85 \\
\hline Users who did not remember seeing our warnings & $44.3 \%(47)$ & $55.7 \%(59)$ & 106 \\
\hline
\end{tabular}

\section{Table 4. Effect of warning recall on resisting our simulated attacks. Of the 200 users in our study, 9 users did not respond to this question.}

was and if you did anything, what you did." Overall, only $6(15 \%)$ challenge questions users and $13(8 \%)$ email users reported seeing anything suspicious during the study. Four of the challenge question users reported that the certificate warning caused their suspicion, but only 1 of those users resisted the attack. ${ }^{8}$ One challenge question user reported that the fact that the attack required her to re-register her machine made her suspicious. The 13 suspicious email users reported the attack instructions as the cause of their suspicion, but only 6 of those users resisted the attack.

\subsection{User reasoning during our attacks}

To help understand users' thought processes during the simulated attack, we showed each user a screenshot of the attack instructions corresponding to her group and asked her: "If you followed the above instructions, explain why. If you chose not follow the instructions, explain why not. If you don't remember this page or what you did, tell us what you don't remember." We did not explicitly identify this as the "attack".

Challenge question users. Among the 38 vulnerable users in the challenge question group, 22 users (58\%) said that they complied with the attack instructions because they thought it was what they needed to do to log in. Representative responses include: "Those were my challenge

\footnotetext{
${ }^{8}$ Most browsers show certificate warnings in popup windows. Firefox 3 and Internet Explorer 7 present full screen interstitial warning pages, but Firefox 3 was not officially released until after we completed our study. Among the 41 challenge question users (who were the only users who saw certificate warnings - see Section 6.6), twenty five used Firefox 1 or 2, two used IE 6, six used IE 7, seven used Safari, and one used Epiphany. Among the 4 users who reported the certificate warning as the cause of their suspicion, three used Firefox 2 and one used IE 7.
}

questions, so I answered them" and "I thought it was procedure to answer these questions." 10 vulnerable users (26\%) viewed the attack as an error that they should try to fix, e.g., "I thought the site's cookie may have been erased which is why it wasn't recognizing my computer, so I answered." 4 vulnerable users $(11 \%)$ trusted the Web site or indicated that since the site was associated with UC-Berkeley, it should be safe. Of the 3 challenge question users who resisted the attack, one user noticed the certificate warning and stopped, and the other two users did not respond to this question.

Email users. Among the 66 vulnerable email users, 26 users $(39 \%)$ complied with the attack instructions because they thought it was what they needed to do to log in. A representative response is "I copy and pasted the link because it said in bold to do so. It seemed like that was what I was supposed to do." 11 vulnerable users (17\%) viewed the attack as an error that they should fix, e.g., "I figured something was wrong with your registration system and thus followed instructions." 20 vulnerable users (30\%) trusted the Web site or indicated that since the site was associated with UC-Berkeley, it should be safe. 8 vulnerable users (12\%) indicated that they complied with our attack instructions because they did not associate much risk with our Web site.

Among the 93 email users who resisted the attack, the responses of 37 users $(40 \%)$ indicated that although they may have recognized the instructions were different from previous registrations, they decided to click the registration link first, despite instructions to the contrary, or did not read the attack instructions carefully. Representative responses include: "I did not follow the instructions because it was easier to just click" and "Usually, in a verification email, you are supposed to click the link." 


\begin{tabular}{|c|c|c|c|c|c|c|c|c|}
\hline \multirow[b]{2}{*}{ Group } & \multirow[b]{2}{*}{$\begin{array}{l}\text { Warnings } \\
\text { in email? }\end{array}$} & \multicolumn{6}{|c|}{ Self-reported effect of warning on user } & \multirow[b]{2}{*}{ Total } \\
\hline & & $\begin{array}{c}\text { Little } \\
\text { or none }\end{array}$ & $\begin{array}{c}\text { Helped } \\
\text { resist atk. }\end{array}$ & $\begin{array}{l}\text { Recalled during } \\
\text { atk. but no help }\end{array}$ & $\begin{array}{l}\text { "Felt } \\
\text { safer" }\end{array}$ & Other & $\begin{array}{l}\text { No } \\
\text { resp. }\end{array}$ & \\
\hline 1 & N/A & 8 & 0 & 0 & 2 & 0 & 3 & 13 \\
\hline 2 & $\checkmark$ & 8 & 7 & 2 & 2 & 4 & 2 & 25 \\
\hline 3 & & 11 & 1 & 1 & 0 & 1 & 2 & 16 \\
\hline 4 & $\checkmark$ & 7 & 2 & 1 & 2 & 5 & 6 & 23 \\
\hline 5 & & 4 & 0 & 0 & 1 & 1 & 2 & 8 \\
\hline Total & & $\overline{c 38}$ & $\overline{10}$ & 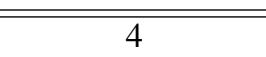 & $\overline{77}$ & $\overline{111}$ & $\overline{15}$ & 85 \\
\hline
\end{tabular}

Table 5. Self-reported effects of our warnings on users who remembered seeing them.

17 resisting users (18\%) indicated that they did not notice the attack instructions. For example, "I never saw these instructions." All except two of these users clicked on the registration link; the other two users timed out the attack.

10 users $(11 \%)$ cited our warnings as helping them resist the attack, e.g., "The website and the email I received were telling me contradicting things so I just went with what the email told me" and "I didn't follow the instructions because they were contradictory to the warnings in the previous email."

We found evidence that 15 users (16\%) initially considered the attack instructions, but eventually gave up because they found them too difficult, decided it was not worth the hassle, or made a mistake in following them. 5 users explicitly indicated this in their responses, e.g., "I did not because it was too much of a hassle" and "I figured I would see if the site would be on track later." The remaining 10 users attempted to follow the attack instructions, but made a "mistake", e.g., they forwarded our welcome email or copy and pasted a previously used registration link. Although we count these users as resisting our attack, they may be more likely to fall for future attacks than other users who resisted.

The responses of 3 resisting users (3\%) were hard to interpret. They stated that they followed the attack instructions, but we have no evidence that they attempted to do so; they all clicked on the link quickly. One possible explanation is that they did not actually notice the attack instructions, but attempted to please us during the survey or avoid appearing as if they disregarded our instructions.

\subsection{Ecological validity}

To evaluate the ecological validity of our study, we sought to determine how much risk users perceived while using our site. Since risk is subjective, we asked each user to tell us the biggest security concerns she has while browsing the World Wide Web and the precautions she takes to protect herself when logging into Web sites. Then, we asked her to rank how often and thoroughly she applies those precautions when logging into the following types of
Web sites: banking, shopping, PayPal, Web email, social networking, and our study site. The answer choices were: "rarely", "sometimes", "usually", "always", and "I don't use this type of Web site". We summarize the responses in Table 6. Users reported that they did not take the same level of precautions on our site as they do with other sites which handle money. Over $64 \%$ of users reported that they at least "usually" take security precautions at those sites, but only $27 \%$ of users said they at least "usually" took precautions at our study Web site. Users more closely associated the risks at our study Web site with a Web email site or social networking site.

In users' responses to other questions, 2 users explicitly mentioned that they took precautions because we had their PayPal email address, e.g., "I wanted to stay secure so that people couldn't come in and take my PayPal account." However, 14 users explicitly mentioned that they considered our study site to be low risk because they felt they had little to lose, e.g., "even if someone had hacked the site, what had I to lose? An experiment account? I was not particularly worried."

\section{Analysis and discussion}

Our warnings were ineffective. Our results suggest that our warnings had little impact users' decisions, even when users had the opportunity to see warnings during the simulated attacks. We found no evidence that including warnings in registration emails helped users resist our attacks. Many users did not remember our warnings or indicated they had little impact on their decisions during the study. Although including contextual warnings in email seemed to improve the likelihood that a user would recall seeing them, we found no evidence that users who recalled seeing our warnings were more likely to resist our attack. Our results are consistent with a recent study by Egelman et al. which suggests that passive warnings such as ours are ineffective in helping users resist attacks [16]. 


\begin{tabular}{|c|c|c|c|c|c|c|}
\hline Site type & Rarely & Sometimes & Usually & Always & Don’t use & No resp. \\
\hline \hline Banking & $10.5 \%(21)$ & $8.5 \%(17)$ & $15.5 \%(31)$ & $55.5 \%(110)$ & $3.5 \%(7)$ & $7.0 \%(14)$ \\
\hline Shopping & $13.0 \%(26)$ & $12.5 \%(25)$ & $26.5 \%(53)$ & $37.5 \%(75)$ & $3.0 \%(6)$ & $7.5 \%(15)$ \\
\hline PayPal & $14.0 \%(28)$ & $9.0 \%(18)$ & $23.0 \%(46)$ & $44.0 \%(88)$ & $3.5 \%(7)$ & $6.5 \%(13)$ \\
\hline Email & $34.0 \%(68)$ & $16.5 \%(33)$ & $18.5 \%(37)$ & $22.0 \%(44)$ & $2.0 \%(4)$ & $7.0 \%(14)$ \\
\hline Social networking & $34.5 \%(69)$ & $21.5 \%(43)$ & $16.0 \%(32)$ & $20.0 \%(40)$ & $1.0 \%(2)$ & $7.0 \%(14)$ \\
\hline \hline Our study Web site & $46.0 \%(92)$ & $18.5 \%(37)$ & $12.5 \%(25)$ & $14.5 \%(29)$ & $1.5 \%(3)$ & $7.0 \%(14)$ \\
\hline
\end{tabular}

Table 6. Risk ratings. This table summarizes how thoroughly and often the users reported applying security precautions when logging into various types of Web sites.

Research from the warning sciences community suggests that if a warning does not sufficiently stimulate users, or if users cannot meaningfully process and apply a warning's message, it will have limited effect [57]. Some responses from email users suggested that we failed to both get their attention and communicate a meaningful message. They assumed our warnings were similar to other "standard" warnings, or our warnings just made them feel our site was generally more secure. For example:

- "I figured it was just standard stuff."

- "It looked like a standard confidentiality issue, so I didn't think of it as anything particularly special."

- "I just chalked it up to general security advice and more or less forgot about it."

- "It made me feel that the website was more secure."

- "This bit of information made me feel like the site was trying to protect my privacy."

- "It did not affect my decisions much, but it did help the validity of the survey."

Lack of user suspicion. Our attacks raised suspicion in only small percentage of users. Many of the other users had alternative interpretations. Some users saw the attack as the result of an error with the Web site, her browser, her computer, or the network, e.g., "I thought that the link was broken." Some users did not view that complying with the attack instructions might be risky, but rather thought it was necessary for their own safety. For example,

- "I followed the instructions because it was for my own safety."

- "I did because I wanted to stay secure so that people couldn't come in and take my PayPal account."

- "The site is verifying I am who I say I am; I never thought of it in terms of me questioning the site's identity."

Some users indicated they did not have a clear understanding of how the registration procedure works and its purpose, and when they would be required to participate in the registration ceremony. For example,
- "I figured that because I switched connections, as I was using Berkeley's wireless as opposed to my dormitory's ethernet Internet, they needed to re-verify my account."

- "I followed the instructions because I assumed my password was wrong so the alternate method of login was by answering the security questions."

- "I figured it had been too many days since I'd signed in."

- "I answered them because I couldn't remember if you guys said that we will randomly be asked to answer them in place of our password and login name."

- "I remembered this page, and I followed the instructions because they are often used to verify a user if a username seems unsafe or has been tampered with."

- "The link in the email contains a data string that, when clicked, changes account details to confirm that that was a valid email address. Security benefits to the user may be minimal."

- "I think it prevents hackers from just creating accounts and using them but they would have to go to the extra step of doing the email registrations."

- "I actually didn't think it had anything to do with the security of my money/identity."

These results are consistent with previous work which suggests that users have a limited understanding of web security mechanisms, Internet social engineering attacks, and effective defense strategies $[13,14,26,58]$. This evidence supports our design principle for conditioned-safe ceremonies that argues designers should not assume users will be able to detect attacks, or sufficiently understand ceremonies to know when they should refrain from participating or perform voluntary defensive actions.

The power of user conditioning and forcing functions. Challenge question based registration conditions users to provide their answers when they are asked their challenge questions. The responses of 58\% of the vulnerable challenge question users indicated that conditioning was the pri- 
mary influence on their decision to comply with the simulated attack's request for their answers. User responses of this type include:

- "I answered the questions because I thought I was being asked to identify myself."

- "I answered it because it was required in order to log in."

- "I wanted to $\log$ in, so I answered the challenge questions."

This supports our hypothesis that challenge questions condition users to answer their challenge questions whenever prompted.

In contrast, the responses of $56 \%$ of email users who resisted the simulated attack suggested that conditioning was a factor in their resistance. The responses of $40 \%$ of resisting email users suggested they may have noticed the attack was somewhat different from a normal registration, but either chose to ignore the attack instructions and click the link, or did not read the attack instructions carefully. User responses of this type include:

- "I didn't follow the instructions because I didn't pay attention to this page (I just followed the usual procedures to register my computer)."

- "I didn't follow the directions because it sounded sketchy and I wanted to see what happened."

- "I must have glossed over the instructions to not click the registration email link, I didn't think there would be two opposing instructions so I just went with the one that was more obvious."

- "I didn't read it carefully, and instinctively clicked on the link in the email."

The responses of $16 \%$ of resisting email users suggested that they probably did not notice any differences between our simulated attack and a normal registration, and proceeded to click on the registration link in the email sent to them. User responses of this type include:

- "I don't remember ever seeing this page, but I think what I might have seen was simply that I thought this page was giving me the same instructions as the first time when I had to register my computer."

- "I don't remember because it has been a hectic week. I just didn't notice."

- "I don't really remember this or I misread it."

- "It's currently 2:20am and I just got back from 5 hours of dance practice. Honestly, I didn't even see the instructions!!! How scary!"

These results suggest that conditioning played a significant role in a large fraction of users' decision making processes during our simulated attacks - to the benefit of email registration, but to the detriment of challenge question based registration.

One factor our study did not control is to what degree challenge questions and clicking on email links had conditioned users prior to participating in our study. Several sites currently implement challenge question based registration [7, 27, 49], and many use challenge questions for password reset. Although we do not know of any sites which implement email registration for machine authentication, many Web sites send an email link to reset a user's password or validate her email address [21]. We did not screen users based on previous exposure to these mechanisms, but we did ask users whether they had previously used them. $80 \%$ of challenge question users and $70 \%$ of email users reported having used the respective mechanisms prior to participating in our study. However, we found no significant correlation between previous exposure to these mechanisms and attack success rate. We leave better understanding of this issue as a subject of future work.

Ecological validity. We asked users to give feedback about their impressions of the study, and their responses suggested that predicting popular movies can be fun and engaging. Some users expressed disappointment that we ended the study before they had the opportunity to make all 7 predictions. Some users admitted they had no idea as to the true purpose of the study, and no user claimed to have figured out that the study was security related. Based on this evidence, we argue the effects of demand characteristics were sharply diminished in our study.

Our study created an experience of risk for some users, but many users indicated that the risk level they associated to our site was roughly equivalent to Web email or social networking sites, and below financial-related sites such as banking or shopping. Some users explicitly stated in their comments that they did not experience much risk during our study, e.g., "And even if someone had hacked the site, what had I to lose? An experiment account? I was not particularly worried." Some users suggested that they felt safer at our site because it was associated with Berkeley, e.g., "I figured that since this was a Berkeley research affiliated website, it would be safe." Creating a significant experience of risk in studies like ours remains a challenge.

Our design attempted to limit the effect of authority figures during the study by conducting it in users' "natural habitats". One concern we had was that users would interpret the attack as instructions from us, the researchers. Although this is similar to the problem users face during a real phishing attack, academic researchers might appear more as an authority figure to a user than, say, a bank. There was evidence that this may have been an issue for some users, e.g., "I followed the instructions because I thought it was a le- 
gitimate set of instructions from respected researchers who could not possibly have a motive to deceive me", but maybe not for others, e.g., "My security is more important to me than their system problems." We did not design our study to evaluate this issue in depth; further research is needed.

\section{User study limitations}

Our study had several limitations. Although we took great efforts to make our study as ecologically valid as possible (while remaining ethical), some users' responses suggested we fell short in some aspects, most notably in simulating the experience of risk in the real world and completely eliminating the influence of authority figures. The size of the compensation may not have been large enough to warrant extra attention, and the fact that our Web site was implicitly associated with UC-Berkeley may have influenced users' decisions.

We acknowledge that there may be more effective attacks against email based registration. One potentially effective attack might be to try to hijack users' email accounts, but we did not implement this attack for ethical reasons. Another type of attack we did not evaluate is a prediction attack. In a prediction attack, the adversary creates the illusion that she can reliably predict the future. Being able to predict the future affords credibility, which an adversary may be able to exploit. If an adversary sends the user an email predicting that she will receive a registration email, but requests that she handles it unsafely, she may be more likely to comply. Stock market scams employing this technique are often effective.

Although our results suggest that the notion of conditioned-safe ceremonies may be useful for helping users resist some types of Internet social engineering attacks, further research is necessary. We acknowledge that it remains to be seen whether the notion of conditioned-safe ceremonies will be more generally applicable to other types of ceremonies, environments, and attack strategies. For example, it may be more challenging to develop conditionedsafe ceremonies to resist attacks whose only goal is to solicit and steal sensitive personal information.

Our study collected a limited amount of information from each user. Since we never met our users, we could not directly observe users' reactions, record comments, or probe for details during the study. Also, since the vast majority of our users were undergraduates at UC-Berkeley, we cannot easily generalize our results to the general population.

\section{Implications and limitations of email based registration}

One might argue that ceremonies that require users to click on email links will train users to click on phishing links and undermine some anti-phishing efforts which caution users to never click on links in email. However, we argue that relying on users to never click on email links is unrealistic. Sending and clicking on links in email is often useful for users, and many password reset and recovery ceremonies currently require users to click on an email link [21]. Some phishing studies suggest that many users regularly click on email links and employ a wide variety of link clicking strategies based on the current task, apparent source of the email, and other contextual cues [13, 15]. It would be a significant challenge to eliminate these practices. We argue that more comprehensive defenses which assume users will click on some email links are more likely to be effective.

Another potential criticism is that email based registration simply shifts many of the security and usability burdens onto email systems. The security of email systems relies on the security of email servers and users' email passwords. This raises several concerns [21]:

- A user might use a weak email password or use the same password for all her accounts.

- Some email providers use weak password reset and recovery mechanisms, such as challenge questions, which may be vulnerable to social engineering and inference attacks.

- Users may view their email accounts as less sensitive than their financial accounts and fail to adequately protect their email passwords. In our study, many users viewed security of their email accounts as having the same level of importance as their accounts at social networking sites, but below their accounts at financial sites.

- Email is often sent over unencrypted connections, and POP and IMAP servers often accept passwords sent over unencrypted connections.

- Employees at businesses or ISPs might have access to their users' email.

- Several users might share a single email account.

- Email delivery is sometimes delayed.

- Spam filters may block legitimate messages.

Although the widespread use of email for password recovery and reset suggests that these issues may be manageable, we should not ignore them. Ideally, we should explore more secure and reliable messaging alternatives for security critical applications. One potential direction is to send registration links to users' mobile phones and develop software which enables easy transfer of the links to users' computers. 


\section{Related work}

Studies which attack users. Security researchers have conducted a number of studies which simulate attacks against users. Several studies have tried to evaluate how well individuals can identify phishing emails and pages [13, 29, 58]. However, these studies do not fully address the design issues we identified in Section 6.1. They were all conducted in a laboratory environment, and the users were either told the purpose of the experiment or asked to roleplay a fictitious identity.

To help create the experience of risk, some laboratory studies have employed deception and required users to participate with their own accounts. Egelman et al. conducted such a study to evaluate the effectiveness of browser phishing warnings [16]. Users made purchases with their own credentials, and the researchers sent the users spear phishing emails related to those purchases which triggered phishing warnings in Firefox and Internet Explorer. Schecter et al. asked real Bank of America SiteKey customers to log into their accounts from a laptop in a classroom [44]. Although SiteKey uses challenge questions, Schecter et al. did not evaluate SiteKey's use of them. Instead, they focused on whether each user would enter her online banking password in the presence of clues indicating her connection was insecure. They simulated site-forgery attacks against each user by removing various security indicators (e.g., her personalized SiteKey image) and causing certificate warnings to appear, and checked if each user would still enter her password. Since SiteKey will only display a user's personalized image after her computer is registered, Schecter et al. first required each user to answer her challenge questions during a "warm-up" task to re-familiarize her with the process of logging into her bank account. No attack was simulated against the users during this task.

Requiring users to use their own accounts is certainly a good start for creating a sense of risk, but the degree to which the academic setting of the physical location of these studies affected the users' evaluation of their actual risk is unclear. Even if the experimenters were not in the same room as the users while they used the computer, the fact that they were nearby may have influenced the users to appear "helpful" and behave with less caution than they normally would.

A few studies have simulated attacks against users in the field without obtaining prior consent. One study at the United States Military Academy at West Point sent cadets a simulated phishing email from a fictitious Colonel "commanding" them to click on a link [19]. Studies by Jagatic et al. [30] and Jakobsson et al. [31] also remotely simulated phishing attacks against users. Although these studies closely simulated real attacks, provided large data sets, and achieved a high level of ecological validity, the absence of prior consent raises ethical issues. After learning that they were unknowing participants in one study, some users responded with anger and some threatened legal action [11]. Also, these studies collected only a limited amount of demographic and behavioral data and did not conduct a exit survey to probe users' decisions.

Email for authentication. Other researchers have proposed leveraging email for authentication [2, 6, 21, 23, 48]. In particular, the design of Simple Authentication for the Web (SAW) by Horst and Seamons is similar to our email registration ceremony [48]. The main difference is that we propose using email only for relatively infrequent machine registrations, i.e., credential initialization, while the SAW authors propose using email authentication as a direct replacement for passwords. In SAW, users receive a fresh email link during each authentication attempt. Also, the SAW authors do not consider social engineering attacks that try to steal authentication links.

User conditioning and education. Previous antiphishing research has attempted to take advantage of user conditioning by using secure attention keys. Two antiphishing tools, PwdHash [43] and Web Wallet [59], employ a secure attention key to create a trusted path between the user and the browser. Although these tools require users to activate the secure attention key before entering any sensitive information, they may be vulnerable to attacks which persuade users to omit the SAK (Section 4.1). A user study of Web Wallet suggests that this attack strategy can be effective [59].

Related to conditioning is training and education. Several researchers have proposed innovative educational methods for teaching users about Internet security and social engineering attacks $[35,45,56]$. Their initial results are promising, and related research suggests that users who better understand Internet risks may be more likely to resist attacks [15]. However, user education may have its limitations. If education is not periodically reinforced, satisficing users may forget or omit defensive habits they have learned. Also, a study consisting of interviews designed to reveal users' decision making strategies for suspicious emails suggests that while users may be able to manage risks they are familiar with, it can be difficult for them to generalize this knowledge to resist unfamiliar attacks [14]. These results suggest that educational approaches may require continual adaptation to address new attacks; otherwise users' defensive strategies may become outdated and ineffective.

\section{Conclusion}

Our study results suggest that 1) ceremonies can affect user behavior, for better or worse, and 2) the resiliency of 
a ceremony to social engineering is related to whether the actions it conditions users to take are safe to perform in the presence of an adversary. These results suggest that conditioned-safe ceremonies may be a useful notion for building ceremonies which resist social engineering attacks. We proposed several design principles for conditioned-safe ceremonies and described one ceremony, email registration, designed according to these principles. Although email registration may be an imperfect approximation of what we would ultimately like out of a conditioned-safe ceremony, we believe it is nonetheless a useful example for exploring and evaluating this notion further. Regardless, the fact that $42 \%$ of email users in our study were vulnerable to our simulated attacks exemplifies the formidable challenge in designing ceremonies to resist social engineering attacks.

\section{Acknowledgments}

This work is supported in part by the TRUST Project (National Science Foundation award number CCF0424422) and the iCAST Project. The conclusions in this paper are our own and do not necessarily reflect those of the NSF, the US Government, or any other funding agency. The authors also thank Rachna Dhamija, Allan Schiffman, Marco Barreno, Adrian Mettler, Monica Chew, AJ Shankar, Bill McCloskey, and the anonymous reviewers for their useful comments.

\section{References}

[1] A. Adams and M. A. Sasse. Users are not the enemy. Communications of the ACM, 42(12):40-46, 1999.

[2] B. Adida. BeamAuth: Two-Factor Web Authentication with a Bookmark. In Proceedings of the Fourteenth ACM Conference on Computer and Communications Security (CCS 07), pages 48-57, October 2007.

[3] C. M. Allwood. Error Detection Processes in Problem Solving. Cognitive Science, 8(4):413-437, 1984.

[4] Anti-Phishing Working Group. http://www. antiphishing.org/.

[5] Anti-Phishing Working Group. Ebay - Update Your Account MITM attack. http: //www . antiphishing . org/phishing_archive/05-03-05_Ebay/ 05-03-05_Eba\%y.html.

[6] D. Balfanz. Usable Access Control for the World Wide Web. In Proceedings of the 19th Annual Computer Security Applications Conference, pages 406-416, December 2003.

[7] Bank of America SiteKey: Online Banking Security. http: //www.bankofamerica/privacy/sitekey/.

[8] Browser market share. http://marketshare. hitslink.com/report.aspx?qprid=0, retrived Sept. 11, 2008.

[9] R. Cialdini. Influence: Science and Practice, 5th edition. Allyn and Bacon, 2008.
[10] T. Close. Waterken YURL. http://www.waterken. $\mathrm{com} / \mathrm{dev} /$ YURL/httpsy/.

[11] C. Corley. Students Go 'Phishing' for User Info. http://www. idsnews.com/news/story. aspx? id $=29400 \&$ comview $=1$.

[12] L. F. Cranor and S. Garfinkel, editors. Security and Usability: Designing Secure Systems That People Can Use. O'Reilly, 2005.

[13] R. Dhamija, J. D. Tygar, and M. Hearst. Why Phishing Works. In Proceedings of the SIGCHI Conference on $\mathrm{Hu}$ man Factors in Computing Systems, pages 581-590, 2006.

[14] J. S. Downs, M. B. Holbrook, and L. F. Cranor. Decision strategies and susceptibility to phishing. In Proceedings of the Symposium on Usable Privacy and Security (SOUPS), pages 79-90, July 2006.

[15] J. S. Downs, M. B. Holbrook, and L. F. Cranor. Behavior response to phishing risks. In APWG 2nd Annual eCrime Researchers Summit, pages 37-44, October 2007.

[16] S. Egelman, L. F. Cranor, and J. Hong. You've Been Warned: An Empirical Study of the Effectiveness of Web Browser Phishing Warnings. In Proceedings of the $\mathrm{CHI}$ 2008 Conference on Human Factors in Computing Systems, 2008.

[17] C. Ellison. Ceremony Design and Analysis. Cryptology ePrint Archive, Report 2007/399, 2007.

[18] C. Ellison, C. Hall, R. Milbert, and B. Schneier. Protecting Secret Keys with Personal Entropy. Future Generation Computer Systems, 16(4):311-318, 2000.

[19] A. J. Ferguson. Fostering E-Mail Security Awareness: The West Point Carronade. EDUCASE Quarterly, 28(1):54-57, 2005.

[20] J. Franklin, V. Paxson, A. Perrig, and S. Savage. An Inquiry into the Nature and Causes of the Wealth of Internet Miscreants. In 14th ACM Conference on Computer and Communications Security (CCS '07), November 2007.

[21] S. Garfinkel. Email-based Identification and Authentication: An Alternative to PKI? IEEE Security \& Privacy Magazine, 1(6):20-26, 2003.

[22] N. Good, R. Dhamija, J. Grossklags, D. Thaw, S. Aronowitz, D. Mulligan, and J. Konstan. Stopping Spyware at the Gate: A User Study of Notice, Privacy and Spyware. In Proceedings of the Symposium on Usable Privacy and Security (SOUPS), pages 43-52, July 2005.

[23] P. Gutmann. Underappreciated Security Mechanisms. http://www.cs.auckland.ac.nz/ pgut001/ pubs/underappreciated.pdf.

[24] P. Gutmann. Security Usability Fundamentals (Draft). http://www.cs.auckland.ac.nz/ pgut001/ pubs/usability.pdf, retrieved Sept. 7, 2008.

[25] C. Haney, W. Banks, and P. Zimbardo. Study of Prisoners and Guards in a Simulated Prison. Naval Research Reviews, 9:1-17, 1973.

[26] A. Herzberg and A. Jbara. Security and Identification Indicators for Browsers Against Spoofing and Phishing Attacks. ACM Transactions on Internet Technology (TOIT), 8(4), September 2008.

[27] ING Direct Privacy Center. https://home. ingdirect.com/privacy/privacy_security. asp?s=newsecurityfe\%ature. 
[28] C. Jackson and A. Barth. ForceHTTPS: Protecting HighSecurity Web Sites from Network Attacks. In Proceedings of the 17th International World Wide Web Conference (WWW 2008), April 2008.

[29] C. Jackson, D. R. Simon, D. S. Tan, and A. Barth. An Evaluation of Extended Validation and Picture-in-Picture Phishing Attacks. In Proceedings of Usable Security (USEC'07), February 2007.

[30] T. Jagatic, N. Johnson, M. Jakobsson, and F. Menczer. Social Phishing. Communications of the ACM, 50(10):94-100, October 2007.

[31] M. Jakobsson and J. Ratkiewicz. Designing Ethical Phishing Experiments: A Study of (ROT13) rOnl Auction Query Features. In Proceedings of the 15th annual World Wide Web Conference ( $W W W 2006$ ), pages 513-522, May 2006.

[32] M. Just. Designing Authentication Systems with Challenge Questions. In L. F. Cranor and S. Garfinkel, editors, Security and Usability: Designing Secure Systems That People Can Use, chapter 8, pages 143-155. O'Reilly, 2005.

[33] C. Karlof, J.D. Tygar, and D. Wagner. A User Study Design for Comparing the Security of Registration Protocols. In First USENIX Workshop on Usability, Psychology, and Security (UPSEC 2008), April 2008.

[34] C. Karlof, U. Shankar, J.D. Tygar, and D. Wagner. Dynamic Pharming Attacks and Locked Same-origin Policies for Web Browsers. In Fourteenth ACM Conference on Computer and Communications Security (CCS 2007), pages 58-72, October 2007.

[35] P. Kumaraguru, Y. Rhee, A. Acquisti, L. F. Cranor, J. Hong, and E. Nunge. Protecting People from Phishing: The Design and Evaluation of an Embedded Training Email System. In CHI '07: Proceedings of the SIGCHI conference on Human factors in computing systems, pages 905-914, 2007.

[36] U. Maimon. Universal Man-in-the-Middle Phishing Kit Why is This Even News? http://www.rsa.com/ blog/entry.asp?id=1160.

[37] S. Milgram. Obedience to Authority: An Experimental View. Harper Collins, 1974.

[38] D. A. Norman. The Design of Everyday Things. Basic Books, 1988.

[39] J. Rasmussen. What Can be Learned from Human Error Reports? In K. D. Duncan, M. M. Gruenberg, and D. Wallis, editors, Changes in Working Life, pages 97-113. Wiley, 1980.

[40] J. Rasmussen. Skills, Rules, and Knowledge: Signals, Signs, Symbols and Other Distinctions in Human Performance Models. IEEE Transactions on Systems, Man, and Cybernetics, 13(3):257-266, 1983.

[41] J. Reason. Human Error. Cambridge University Press, 1990.

[42] A. Reber. Penguin Dictionary of Psychology, 2nd Edition. Penguin Books, 1995.

[43] B. Ross, C. Jackson, N. Miyake, D. Boneh, and J. C. Mitchell. Stronger Password Authentication Using Browser Extensions. In Proceedings of the 14th USENIX Security Symposium, pages 17-32, August 2005.

[44] S. Schechter, R. Dhamija, A. Ozment, and I. Fischer. Emperor's New Security Indicators: An Evaluation of Website Authentication and the Effect of Role Playing on Usability Studies. In Proceedings of the 2007 IEEE Symposium on Security and Privacy, pages 51-65, May 2007.
[45] S. Sheng, B. Magnien, P. Kumaraguru, A. Acquisti, L. F. Cranor, J. Hong, and E. Nunge. Anti-Phishing Phil: The Design and Evaluation of a Game That Teaches People Not to Fall for Phish. In Proceedings of the Symposium on Usable Privacy and Security (SOUPS), pages 88-99, July 2007.

[46] C. Soghoian and M. Jakobsson. A Deceit-Augmented Man in the Middle Attack Against Bank of America's SiteKey Service. http://paranoia.dubfire.net/2007/ 04 /deceit-augmented-man-in-middle-atta\% ck.html, April 2007.

[47] R. Thomas and J. M. (a.k.a. Team Cymru). The Underground Economy: Priceless. ;login: The USENIX Magazine, 31(6):7-16, December 2006.

[48] T. W. van der Horst and K. E. Seamons. Simple Authentication for the Web. In 3rd International Conference on Security and Privacy in Communication Networks (SecureComm), September 2007.

[49] Vanguard Security Center. https : / / www . vanguard. $\mathrm{com} /$.

[50] W. A. Wagenaar and J. Groeneweg. Accidents at Sea: Multiple Causes and Impossible Consequences. International Journal of Man-Machine Studies, 27(5/6), Nov/Dec 1987.

[51] Washington Post. Citibank Phish Spoofs 2-Factor Authentication. http://blog.washingtonpost. com/securityfix/2006/07/citibank_phish_ spoof $\%$ s_2factor_1.html.

[52] Washington Post. Not Your Average Phishing Scam. http://blog.washingtonpost.com/ securityfix/2007/01/not_your_average_ amazon_phishi.html.

[53] R. West. The Psychology of Security: Why Do Good Users Make Bad Decisions? Communications of the ACM, 51(4):34-40, April 2008.

[54] T. Whalen and K. M. Inkpen. Gathering Evidence: Use of Visual Security Cues in Web Browsers. In Proceedings of Graphics Interface 2005, pages 137-144, May 2005.

[55] A. N. Whitehead. Introduction To Mathematics. Williams and Northgate, 1911.

[56] A. Whitten and J.D. Tygar. Safe Staging for Computer Security. In Workshop on Human-Computer Interaction and Security Systems, April 2003.

[57] M. S. Wogalter, editor. Handbook of Warnings. Lawrence Erlbaum Associates, 2006.

[58] M. Wu, R. C. Miller, and S. Garfinkel. Do Security Toolbars Actually Prevent Phishing Attacks? In Proceedings of the SIGCHI Conference on Human Factors in Computing Systems, pages 601-610, 2006.

[59] M. Wu, R. C. Miller, and G. Little. Web Wallet: Preventing Phishing Attacks by Revealing User Intentions. In Proceedings of the Symposium on Usable Privacy and Security (SOUPS), pages 102-113, July 2006.

[60] K.-P. Yee. Guidelines and Strategies for Secure Interaction Design. In L. F. Cranor and S. Garfinkel, editors, Security and Usability: Designing Secure Systems That People Can Use, chapter 13, pages 247-273. O'Reilly, 2005.

[61] J. Youll. Fraud Vulnerabilities in SiteKey Security at Bank of America. cr-labs.com/publications/ SiteKey-20060718.pdf, July 2006. 